\title{
Adaptive State-of-Charge Indication System for Li-ion Battery-Powered Devices
}

\author{
V. Pop*, H.J. Bergveld**, D. Danilov***, P.P.H. Notten***,****, P.P.L. Regtien*****
}

An algorithm that combines adaptive system with Electro-Motive-Force (EMF) measurements during equilibrium and current measurement and integration during (dis)charge states has been implemented in a real-time SoC evaluation system. During the discharge state also the effect of the overpotential is considered.

The overpotential model includes a variety of parameters that are changing during cycling of the battery. Adaptive methods for the battery maximum capacity and for the overpotential model parameters are presented in this paper. The final aim is to predict the SoC and the remaining run-time of a Li-ion battery within $1 \%$ inaccuracy under all realistic user conditions.

Keywords: portable energy; Li-ion batteries; State-of-Charge (SoC); State-of-Health (SoH).

\section{INTRODUCTION}

Battery-powered devices have become ubiquitous in modern society. The recent rapid expansion in the use of portable devices (e.g. portable computers, cellular phones, personal data assistants, etc) and electrical vehicles creates a strong demand for fast deployment of battery technologies at an unprecedented rate. In these applications the request for an accurate and reliable State-of-Charge (SoC) indicator system is clear. SoC is defined as the percentage of the maximum possible charge that is present inside a rechargeable battery [1]-[5]. Accurate SoC indication is difficult due to spread in battery and user behaviour, aging, large range of load current and temperature and different batteries chemistries.

During the lifetime of a battery, its performance or "health" tends to deteriorate gradually due to irreversible physical and chemical changes that take place with usage and with age until eventually the battery is no longer usable [1]. Aging of the battery is a complex process that involves many parameters of the battery, e.g. impedance and capacity [4]. The State-of-Health $(\mathrm{SoH})$ is an indication of the point that has been reached in the life cycle of the battery and a measure of its condition relative to a fresh battery. In order to deal with the aging effect an

*Holst Centre / IMEC-NL, High Tech Campus 48, 5656AE, Eindhoven, the Netherlands, e-mail: Valer.Pop@imec-nl.nl

**NXP Research, High Tech Campus 5, 5656AE, Eindhoven, the Netherlands, email: HenkJan.Bergveld@nxp.com

***Eindhoven University of Technology, P.O. Box 513, 5600MB, Eindhoven, the Netherlands, Danilov@eurandom.tue.nl

****Philips Research Laboratories, High Tech Campus 4, 5656AE, Eindhoven, the Netherlands, email: Peter.Notten@philips.com

$* * * * *$ University of Twente, P.O. Box 217, 7500AE, Enschede, the

Netherlands, email: p.p.1.regtien@utwente.nl adaptive system has to be used for SoC indication. For a good overview of existing adaptive systems for SoC indication the reader is referred to [4].

This paper is organized as follows. General issues concerning the battery aging effect are given in section 2. The State-of-Charge (SoC) algorithm is introduced in section 3. Section 4 focuses on overpotential measurement and modelling methods. New adaptive system for the battery maximum capacity and overpotential are presented in section 5. Section 6 focuses on the obtained results. Finally, section 7 presents the concluding remarks and future work.

\section{GENERAL ISSUES CONCERNING THE BATTERY AGING PROCESS}

A Li-ion battery may loose performance during the battery lifetime due to the increase in the impedance and overpotential or/and due to the decrease in the maximum capacity $\left(Q_{\max }\right)$ [1]. The operational conditions determine whether an aged battery can still deliver acceptable remaining run-times $\left(t_{r}\right)$ to the user. A battery with a low $Q_{\max }$ or a high overpotential may still deliver acceptable run-times for the portable devices that require low discharge $\mathrm{C}$-rate currents, e.g. a Compact Disk (CD) player or a wireless mouse. The portable devices that require high discharge C-rate currents, e.g. mobile phones, digital cameras or Electrical Vehicles (EV's), on the other hand, will deliver shorter remaining run-times. As a result the battery must be recharged more often. This will lead to even more wear-out.

The changing rate in the battery overpotential and maximum capacity is strongly dependent on the operational conditions. High C-rates for the charge/discharge currents and high temperatures and voltage levels during the battery charging will 
speed-up the changing rate of these two battery characteristics. To illustrate these phenomena the discharge battery capacity $\left(Q_{d}\right)$ is plotted for two different operational conditions as function of the cycle number in Fig. 1. In both examples the discharge battery capacity has been inferred by means of coulomb counting [1]-[8] from a complete discharge step at $0.5 \mathrm{C}$-rate current.

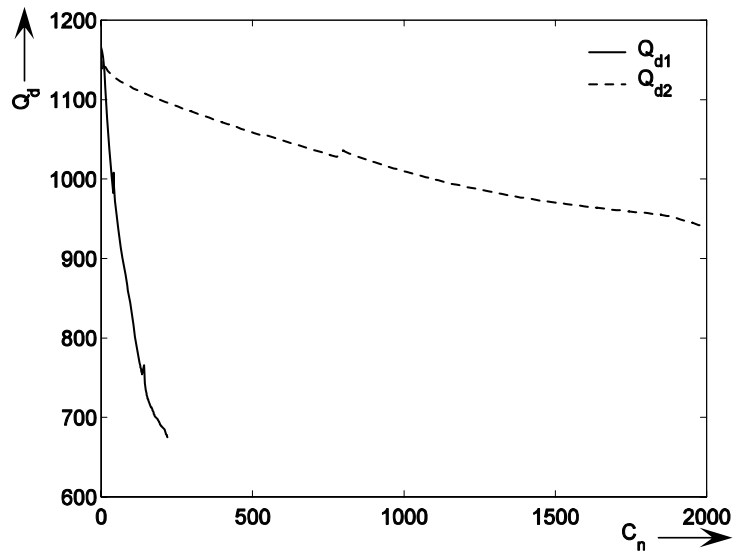

Fig. 1. Decrease of the discharge capacity $Q_{d}$ [mAh] as function of the operational conditions. The horizontal axis shows the cycle number $C_{n}$.

The decrease in discharge capacity can be expressed as

$$
Q_{d i}^{j}[\%]=100\left(1-\frac{Q_{d i}^{j}}{Q_{d i}^{1}}\right)
$$

where $Q_{d i}^{j}[\%]$ denotes the decrease in $Q_{d}[\mathrm{mAh}]$ after $j$ cycles and $i$ the battery number.

Fig. 1 shows that the decrease in $Q_{d}$ strongly depends on the operational conditions, i.e. on the charge/discharge C-rate current, the charge voltage limit and temperature. For instance, during the operational conditions performed for the battery with $Q_{d l}$ (continuous line in Fig. 1) the battery has been charged by means of the Constant-CurrentConstant-Voltage (CCCV) method [1] until $4.3 \mathrm{~V}$ at $25^{\circ} \mathrm{C}$. During the $\mathrm{CC}$ step a $4 \mathrm{C}$-rate current has been applied. It follows from Fig. 1 that $Q_{d 1}$ had a value of $675 \mathrm{mAh}$ after 220 cycles, whereas after the first cycle it was $1165 \mathrm{mAh}$. It can be concluded from this example that after 220 cycles $Q_{d l}$ value decreases with about $42 \%$ when compared to $Q_{d 1}$ value after the first cycle.

In the second case, (dashed line in Fig. 1) the battery has been partially charged/discharged between $30 \%$ and $70 \%$ SoC with $0.5 \mathrm{C}$-rate current at $25^{\circ} \mathrm{C}$. It follows from Fig. 1 that $Q_{d 2}$ had a value of $935 \mathrm{mAh}$ after 2000 cycles, whereas the value of $Q_{d 2}$ after the first cycle was $1150 \mathrm{mAh}$. So, $Q_{d 2}$ drops by $19 \%$ in 2000 cycles with respect to $Q_{d 2}$ value after the first cycle.
It should be noted that the decreases in the discharge capacity illustrated in Fig. 1 is a result of two combined battery processes, e.g. an increase in the battery overpotential and a decrease in the battery maximum capacity. These processes are accelerated when the battery is fully charged with high C-rate currents, until high voltage levels and at high temperatures [9]. Due to the increase in the battery overpotential at the end of the dis(charging) less capacity will be removed(put) from(in) the battery. Due to the decrease of the battery maximum capacity less capacity will be removed(put) from(in) the battery during dis(charging).

The examples discussed in this section show that the aging of the battery is a complex process that involves many battery parameters, such as impedance and capacity, where the most important characteristic seems to be the battery capacity. So, for a more accurate determination of the remaining run-time the variation of these two parameters should be taken into account. To understand how this is realized, first the basic methods for SoC determination are discussed.

\section{STATE-OF-CHARGE INDICATION}

\subsection{State-of-Charge Indication Methods}

There are several practical methods available for SoC indication. From these methods, the two best known are direct measurement and book-keeping [1]-[4]. The direct-measurement method is based on the measurement of battery variables such as the battery voltage $(V)$, the battery impedance $(Z)$ and the voltage relaxation time $(\tau)$ after application of a current step. A particular method is based on the measurement of EMF, which is the sum of the equilibrium potentials of the electrodes of the battery [1], [9]. EMF can be directly translated into a SoC value using a predetermined function. EMF of a Li-ion battery is observed to be a good measure for a battery SoC. It has been demonstrated that the relationship between EMF and SoC does not change during cycling of the battery, if SoC is expressed in relative capacity [1].

The book-keeping method is based on integration of the current $(I)$ flowing into and out of the battery, often referred to as coulomb counting [1]. However, the battery does not behave like a linear capacitor. This implies that not all charge supplied to the battery can actually be retrieved under all conditions. Therefore, in addition to these coulomb counting data other relevant battery data such as discharge efficiency, self-discharge rate of the battery, temperature and history (e.g. cycle life) will be used as input for the book-keeping system [1].

The main problem in designing an accurate SoC indication system is the unpredictability of both battery and user behaviour. In this situation an adaptive system has to be used. An innovative 
adaptive system for SoC indication will be described in section 5 .

An new algorithm that combines adaptive system with Electro-Motive-Force (EMF) measurements during equilibrium and current measurement and integration during charge and discharge states has been developed [1]-[5]. During the discharge state, apart from simple coulomb counting, also the effect of the overpotential is considered.

\subsection{Implementation Aspects of the State-of-Charge Algorithm}

In order to check the SoC and remaining run-time accuracy the new SoC algorithm has been implemented in a real-time $\mathrm{SoC}$ evaluation system, which operates in six different states: initial state, standby state, backlight-on state, transitional state, charge state and discharge state. The state diagram illustrating the basic structure of the real-time SoC evaluation system is shown in Fig. 2, where the backlight-on state has not been included.

Each time the SoC evaluation system is switched-on, it starts from the initial state (see Fig. 2). SoC in the initial state is determined by means of voltage $(V)$ and temperature $(T)$ measurements and the stored EMF model $\left(E M F_{m}\right)$. Dependent on whether the battery is charged, discharged or in equilibrium, the SoC evaluation system then switches to the appropriate state.

In the standby state hardly any current is drawn from the battery. In this situation the battery is in equilibrium after full relaxation. SoC in the standby state $\left(S o C_{s}\right)$ is determined by means of $V$ and $T$ measurements and the stored $E M F_{m}$.
The current in the standby state is only a few $\mathrm{mA}$, e.g. 1 $\mathrm{mA}$ current in the SoC evaluation system described in this section, which is lower than the current limit $I_{\text {lim }}$ defined in the SoC evaluation system of $10 \mathrm{~mA}$ (see Fig. 2 ). For this very low standby current value, the battery voltage is very close to the EMF value under the condition that the voltage is stable. Therefore, in order to allow the SoC evaluation system to change to this state, the condition of a stable voltage has to be met.

In the backlight-on state a small negative current, e.g. $6 \mathrm{~mA}$ current in the SoC evaluation system described in this section, is drawn from the battery. This situation occurs when the user activates the screen of the SoC evaluation system. Because the current is still below $I_{\text {lim }}$, SoC is still determined by means of $V$ and $T$ measurements and the stored $E M F_{m}$. The SoC evaluation system remains in this state for about 5 seconds (an arbitrary chosen value) before it switches automatically into the standby state. During these 5 seconds, all other transitions to the charge or discharge states remain possible.

The transitional state occurs when the system changes from either the charge or discharge state to the standby state (see Fig. 2). In this state a small negative current, e.g. $1 \mathrm{~mA}$ current in the SoC evaluation system described in this section, is drawn from the battery. Because the battery voltage is not stable SoC in the transitional state is determined by means of coulomb counting (cc) [1]-[8]. In this state it is determined whether the battery voltage reached a stable value, i.e. EMF and the system is allowed to enter into the standby state.

In the discharge state, the battery is discharged and a negative current $I_{d}$ larger in modulus than $I_{\text {lim }}$ flows out of the battery (see Fig. 2).

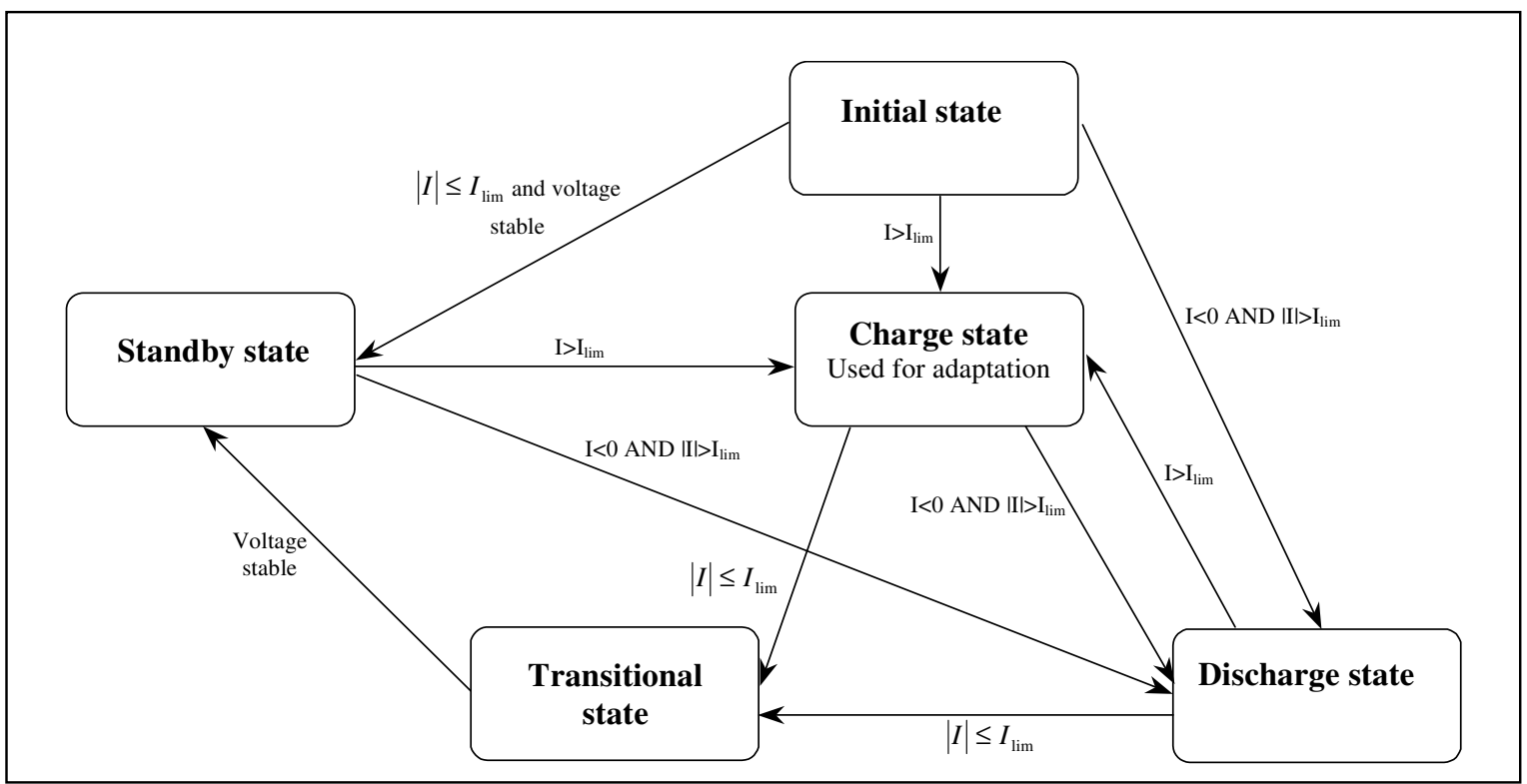

Fig. 2. State diagram of the real-time SoC evaluation system. 
SoC in the discharge state $S o C_{d}$ is determined by means of cc. In addition to $S o C_{d}$, the remaining run-time available under the discharge conditions is also calculated during the discharge state. As a result in addition to simple cc also the effect of the battery overpotential is considered for the remaining run-time calculation. The predicted battery overpotential at the point where the battery is considered empty is translated into a $\mathrm{SoC}$ percentage value $\mathrm{SoC}_{l}[\%]$ by using the EMF model.

As a result $t_{r}[\mathrm{~min}]$ is inferred from $\mathrm{SoC}_{d}[\%], \mathrm{SoC}_{l}$ [\%], $Q_{\max }[\mathrm{mAh}]$ and $I_{d}[\mathrm{~A}]$ according to

$$
t_{r}[\mathrm{~min}]=\frac{0.06 \frac{Q_{\max }}{100}\left(S_{o} C_{d}-S o C_{l}\right)}{I_{d}}
$$

Apparently, in order to enable accurate remaining run-time prediction accurate battery overpotential modelling is necessary. For this reason, an overpotential model will be developed in the next section.

In the charge state, a charger is connected to the battery and a positive current larger than $I_{\text {lim }}$ flows into the battery (see Fig. 2). The SoC in the charge state is determined by means of cc. The stable conditions of the charge state are exploited to adapt $Q_{\max }$ with the aging effect. The $Q_{\max }$ adaptation method will be explained in section 5 .

In summary, in which state the real-time SoC evaluation system is operating depends on the value and sign of the current, which is flowing into or out of the battery and whether the battery voltage is stable or not.

\section{OVERPOTENTIAL MODELLING AND SIMULATION RESULTS}

The overpotential is the difference between EMF and the voltage during charging/discharging the battery. As soon as the discharge or charge process starts, the overpotential subtracts from or adds to EMF [1]-[5]. Due to this overpotential, the battery voltage during (dis)charge is (lower)higher than EMF. Overpotential modelling and simulation results will be presented below.

\subsection{Overpotential Modelling}

The overpotential, in which the ohmic, kinetic, diffusion overpotential and the increase of the diffusion overpotential when the battery becomes empty are considered, is modelled as follows

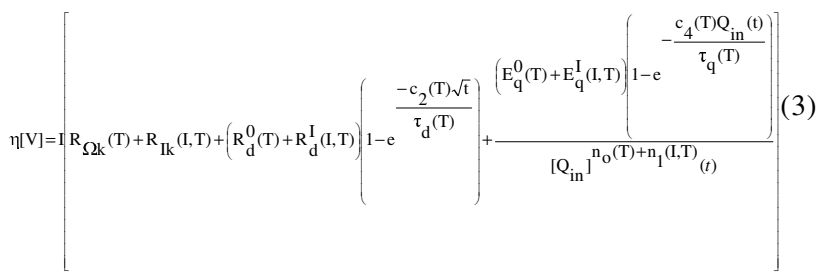

where $I[\mathrm{~A}]>0$ for discharge, $I[\mathrm{~A}]<0$ for charge,
$R_{\Omega k}(T)[\Omega], R_{I k}(I, T)[\Omega]=c_{0}(T)\left[\Omega A^{-1}\right] I[A]$, denote the contributions of the "ohmic" and "kinetic" resistance, $R_{d}^{0}(T)[\Omega]$ and $\mathrm{R}_{\mathrm{d}}^{\mathrm{I}}(\mathrm{I}, \mathrm{T})[\Omega]=\mathrm{c}_{1}(\mathrm{~T})[\mathrm{V}] / \mathrm{I}[\mathrm{A}]$, denote the contributions of the "diffusion" resistance, $c_{2}(T){ }^{\left[\mathrm{s}^{1 / 2}\right]}$ is a constant, $\tau_{d}(T)[s]$ denotes the "diffusion" time constant, $n_{0}(T)$ (dimensionless) and $\mathrm{n}_{1}(\mathrm{I}, \mathrm{T})=\mathrm{c}_{5}(\mathrm{~T})[\mathrm{A}] / \mathrm{I}[\mathrm{A}]$ are parameters related with the magnitude of the diffusion overpotential, $E_{q}^{0}(T)\left[\mathrm{J} \mathrm{A}^{-1}\right]$ and $\mathrm{E}_{\mathrm{q}}^{\mathrm{I}}(\mathrm{I}, \mathrm{T})\left[\mathrm{JA}^{-1}\right]=\mathrm{c}_{3}(\mathrm{~T})[\mathrm{J}] / \mathrm{I}[\mathrm{A}]$, denote the amount of the energy that cannot be obtained from the battery when the current $I$ increases, $Q_{i n}(t)[\mathrm{C}]$ denotes the charge present in the battery at the time $t[\mathrm{~s}]$ and $c_{2}\left[s^{1 / 2}\right]=c_{4}\left[A^{-1}\right]=1$ numerically. Finally, $\tau_{q}(T)$ is a time constant associated with the increase in overpotential in an almost empty battery in [s].

\subsection{Simulation Results of the Overpotential Model}

The results of the mathematical implementation of the overpotential function will be presented in this section. The US18500G3 Li-ion battery has been used throughout the experiments. The overpotential model described by Eq. (3) needs to be fitted to measured overpotential curves.

The measured and fitted overpotential (Eq. (3)) at four different discharge current rates as a function of the relative SoC are compared in Fig. 3. The measurements have been carried out at $25^{\circ} \mathrm{C}$. In order to better show the closeness between measured and fitted data, the difference between the measured and fitted overpotentials are plotted in Fig. 4.

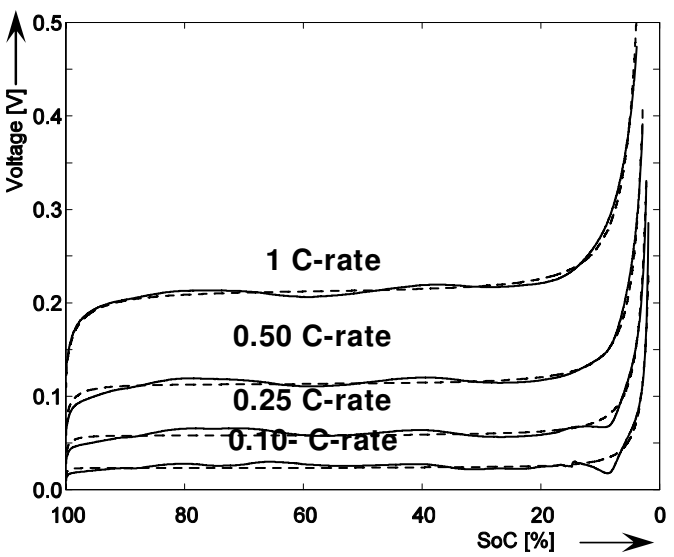

Fig. 3. The measured (continuous line) and the fitted (dashed line) overpotential curves obtained at different $\mathrm{C}$-rate currents and at $25^{\circ} \mathrm{C}$ during discharge as function of SoC [\%] normalized to the maximum capacity. 
It can be concluded from Figs. 3 and 4 that the maximum difference between the measured and the fitted overpotential occurs for a $0.1 \mathrm{C}$-rate discharge current at low SoC. In this situation, at $1.85 \%$ SoC the obtained difference has a value of around $57 \mathrm{mV}$. This voltage error corresponds to a low SoC error $\left(\mathrm{SoC}_{e}\right)$, i.e. $\mathrm{SoC}_{e}=0.4 \%$, which can still be removed from the battery. The remaining run-time error is found from the State-of-Charge error by

$$
t_{r}[\mathrm{~min}]=\frac{\operatorname{SoC}_{e}[\%]}{100} \frac{1}{C_{d}} 60
$$

where $\mathrm{SoC}_{e}$ denotes the error in SoC in [\%] and $C_{d}$ the discharge current in C-rate.

It follows that even for a new battery, at $25^{\circ} \mathrm{C}$ and at $0.1 \mathrm{C}$-rate mean discharge current the $\mathrm{SoC}$ system will indicate 2.4 minutes more remaining run-time than in the real case. However, it follows from Fig. 4 that in the majority of cases a very good fit (under $10 \mathrm{mV}$ difference) between the two curves is obtained. Therefore, the overpotential evolution can be accurately modelled even for low SoC. This part of the curve is practically most important, since it describes the amount of remaining charge for a given $\mathrm{C}$-rate current. It can be concluded that the overpotential implementation results in a final accuracy of better than 2.4 minutes in remaining run-time.

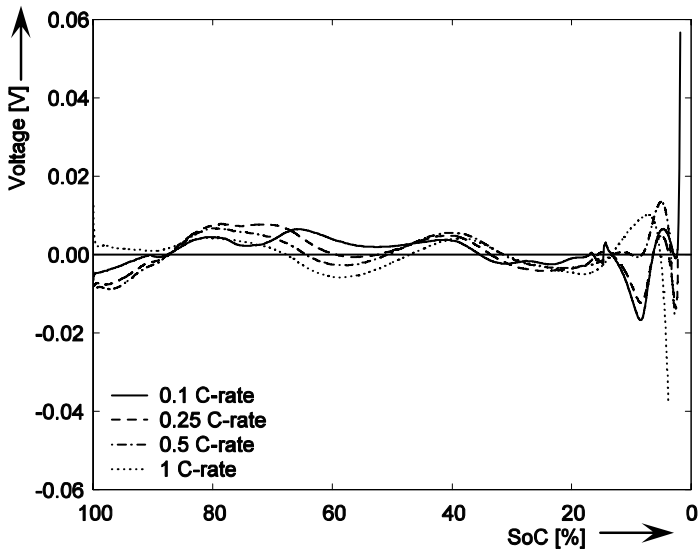

Fig. 4. The difference between the measured and the fitted overpotential obtained during discharging at different currents at $25^{\circ} \mathrm{C}$ (see also Fig. 3). SoC [\%] is normalized with respect to the maximum capacity.

The values of the overpotential model parameters have been determined independently from the C-rate current and are summarized in Table 1.

In the remainder of this section it will be shown that the set of parameters retrieved from the discharge overpotential modelling works well also for the charging process. Fig. 5 illustrates the measured and the fitted overpotential retrieved at different discharge currents and at charging (1 C-rate). Fig. 5 shows that the charge and discharge overpotentials retrieved at 1 C-rate current are symmetrical. Therefore, the overpotential evolution under charging conditions can be accurately modelled especially in the symmetrical part between 20 and $80 \%$ SoC. More information on the overpotential symmetry will be given in the next section.

Table 1 . The battery overpotential model parameter values retrieved at $25^{\circ} \mathrm{C}$.

\begin{tabular}{|c|c|c|}
\hline Parameter & Value $\left(25^{\circ} \mathrm{C}\right)$ & Unit \\
\hline$R_{\Omega k}$ & $1.1110^{-1}$ & {$[\Omega]$} \\
\hline$c_{0}$ & $-2.0210^{-2}$ & {$\left[\Omega \mathrm{A}^{-1}\right]$} \\
\hline$R_{d}^{0}$ & $9.2410^{-2}$ & {$[\Omega]$} \\
\hline$c_{1}$ & $-4.7610^{-4}$ & {$[\mathrm{~V}]$} \\
\hline$c_{2}$ & 1.00 & {$\left[\mathrm{~s}^{1 / 2}\right]$} \\
\hline$\tau_{d}$ & 1.05 & {$[\mathrm{~s}]$} \\
\hline$E_{q}^{0}$ & $2.2110^{3}$ & {$\left[\mathrm{~J} \mathrm{~A}^{-1}\right]$} \\
\hline$c_{3}$ & $5.5710^{2}$ & {$[\mathrm{~J}]$} \\
\hline$c_{4}$ & 1.00 & {$\left[\mathrm{~A}^{-1}\right]$} \\
\hline$\tau_{q}$ & $5.4210^{-2}$ & {$[\mathrm{~s}]$} \\
\hline$n_{0}$ & 1.81 & {$[1]$} \\
\hline$c_{5}$ & $1.0610^{-2}$ & {$[\mathrm{~A}]$} \\
\hline
\end{tabular}

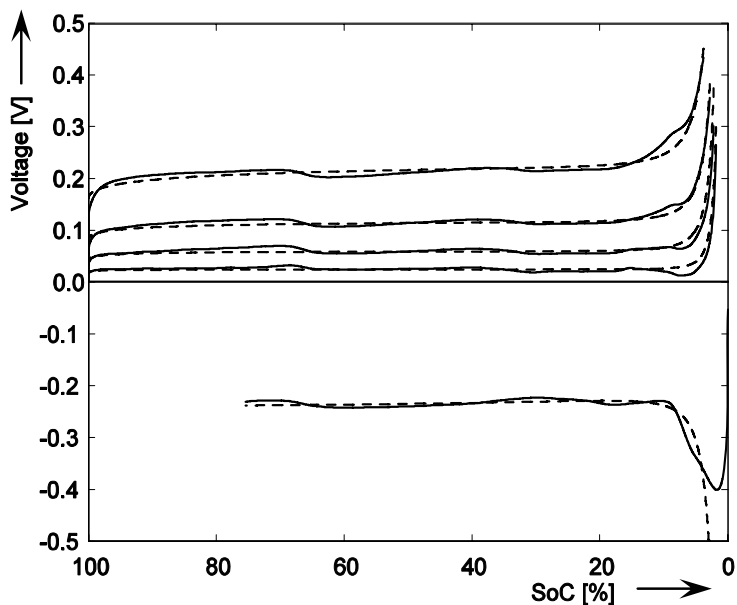

Fig. 5. The measured (continuous line) and the fitted (dashed line) overpotential curves obtained at different discharge currents and at charging $\left(1 \mathrm{C}\right.$-rate) at $25^{\circ} \mathrm{C}$.

Since the overpotential increases for old cells the inaccuracy of the indicated $\mathrm{SoC}$ in these situations will be higher. In conclusion, in order to enable accurate SoC indication the overpotential model parameters should be adapted for aging effects. 


\section{ADAPTIVE SYSTEMS}

In order to enable accurate SoC indication a new adaptive system for the battery maximum capacity and overpotential model will be developed in this section. This adaptive system is necessary since as shown in section 2 the battery maximum capacity and overpotential behaviour will change over time because of aging processes (see Fig. 1).

\subsection{Maximum Capacity}

As shown in section 2 any battery will lose capacity during cycling (see Fig. 1). In order to enable accurate SoC and $t_{r}$ calculation and to improve the SoC evaluation system capability to cope with the aging effect, a simple method for updating the maximum capacity $\left(Q_{\max }\right)$ taking capacity loss into account, will be presented. In this method the stable conditions of battery charging are exploited in order to adapt $Q_{\max }$ for the aging effect. Fig. 6 shows how the maximum capacity is being updated. It is necessary for the system to run through a sequence of states: standby state, charge state, transitional state and standby state (see Fig. 6). A new value of $Q_{\max }$ is determined as follows

$$
Q_{\max }=\frac{100}{S o C_{s f}-S o C_{s i}} Q_{c h}
$$

where $S o C_{s i}$ and $S o C_{s f}$ denote the initial and final State-of-Charge in the standby state in [\%], SoC $C_{s}$, and $Q_{c h}$ denotes the amount of charge flowing into the battery during the charge state in [mAh].

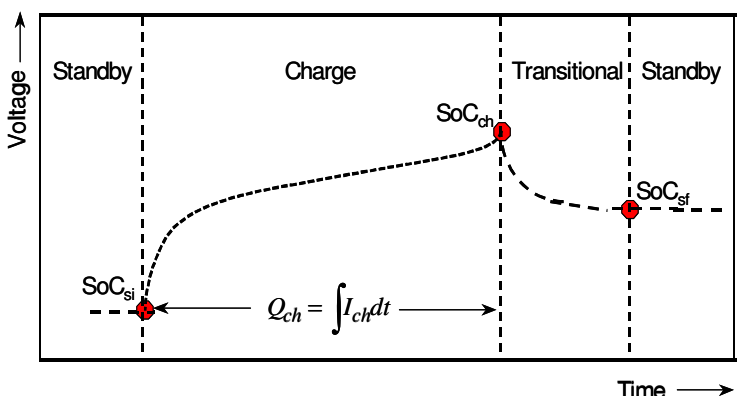

Fig. 6. Schematic representation of the method for updating $Q_{\max }$ taking capacity loss into account.

\subsection{Overpotential Adaptive System}

In addition to the change in the maximum capacity the overpotential development of the battery will also change over time. A simple reason for this is the fact that the series resistance of the electrodes will increase over time. In addition, other contributions to the overpotential related to chemical behaviour of the battery will also change during the lifetime of the battery [1]. When the change in overpotential behaviour is not taken into account the overpotential model described by Eq. (3) will have less and less accuracy when the battery ages.

The main problem with overpotentials is that they cannot be measured directly [1]. When the battery voltage is measured and EMF is known an estimate of the overpotential can, however, be derived. A remaining difficulty is the fact that the overpotential depends on many factors, including SoC, current, temperature, time, spread and age of the battery [1]. Therefore, updating should be initiated

when most of these variables are kept constant. A new method for adapting the battery overpotential with the aging effect will be presented in this section. The symmetry in the overpotential will, in addition, be used for this adaptive system. More information regarding the implementation aspects and test results of this method will be presented in section 6 .

The proposed updating mechanisms for the overpotential model parameters take advantage of the fact that updating is performed during charging. The current is constant in the CC mode and the temperature can also be considered constant because in most cases the charger will be used in-house, where temperature variations are limited. Moreover, during normal CC charging the charge current is not interrupted, so after the overpotentials have been built-up at the initial stages of charging the time variable does not play a dominant role.

The concept of the overpotential adaptive method is as follows. The overpotential model parameters for a fresh battery $\left(\eta^{f}\right)$ are retrieved by fitting the overpotential model (see Eq. (3)) on measured discharge overpotentials $\left(\eta_{d}^{f}\right)$ retrieved at four C-rate currents together with the measured charge overpotential $\left(\eta_{c h}^{f}\right)$ retrieved during the $\mathrm{CC}$ charge mode (see section 4$). \eta_{c h}$ can be measured for each SoC during the CC charge mode, by subtracting the EMF value from the measured battery voltage value. SoC in percentage can be used to assess the EMF value during the $\mathrm{CC}$ charge mode using the SoC-EMF relationship described in [8]. During the battery lifetime the charge overpotential for an aged battery $\left(\eta_{c h}^{a}\right)$ can be continuously measured by using the above-described $\eta_{c h}$ measurement method. As a result, a ratio at the same SoC, C-rate current and temperature can be calculated between the fresh and the aged battery charge overpotentials. As shown in section 4 the charge and discharge overpotentials are symmetrical with respect to the horizontal axis. For this reason, the ratio $\eta_{c h}^{a} / \eta_{c h}^{f}$ determined for the charge battery overpotential work well also for the discharge battery overpotential. As a result the battery discharge overpotential for an aged battery $\left(\eta_{d}^{a}\right)$ can be determined by means of Eq. (6). 


$$
\eta_{d}^{a}=\eta_{d}^{f} \frac{\eta_{c h}^{a}}{\eta_{c h}^{f}}
$$

It should be noted that the charge and discharge overpotentials for the fresh and for the aged battery must be determined at the same SoC, C-rate current and temperature.

\section{RESULTS WITH THE ADAPTIVE SOC EVALUATION SYSTEM}

A set of tests has been carried out with the described real-time SoC evaluation system in order to demonstrate the SoC algorithm accuracy and the SoC evaluation system validity. A US18500G3 Li-ion battery from Sony has been used throughout the tests. At the time of testing, the battery had approximately 600 discharge/charge cycles in its history. The tests have been carried out at full charge/discharge cycles at different constant currents at $25^{\circ} \mathrm{C}$. A value of 1176 $\mathrm{mAh}$ for the maximum capacity and overpotential parameters values retrieved for a fresh battery (see Table 1) have been stored into the SoC evaluation system at the beginning of the tests. During the charge cycles the battery has always been fully charged until $4.2 \mathrm{~V}$ with the normal CCCV charging method at 0.5 $\mathrm{C}$-rate current in the $\mathrm{CC}$ mode. In the $\mathrm{CV}$ mode the voltage has been kept constant at $4.2 \mathrm{~V}$ until the current reached a $0.05 \mathrm{C}$-rate value. Each period of charging has been followed by a rest period of 0.5 hours. After this rest period a discharge step at $0.5 \mathrm{C}$-rate current has been applied until the battery voltage reached $3 \mathrm{~V}$. This procedure has been repeated for a $0.75 \mathrm{C}$ discharging current. During the charge cycles a new value for the battery maximum capacity and a value for the ratio $\eta_{c h}^{a} / \eta_{c h}^{f}$ have been obtained by means of the adaptive methods described in section 5. As a result, a new maximum capacity of $1108 \mathrm{mAh}$ and a value of 1.4 for the ratio $\eta_{c h}^{a} / \eta_{c h}^{f}$ have been measured and programmed into the SoC evaluation system. The above-described measurements have been repeated, with the newly determined parameter values for different constant discharge C-rate currents $(0.1,0.25,0.5,0.75$ and $1 \mathrm{C})$.

In Table 2 the experimental results are summarised. A distinction has been made in this table between the results retrieved without using an adaptive system and the results retrieved by using the newly developed adaptive system. The discharge $\mathrm{C}$-rate current at which these tests have been carried out is given in column 1 . SoC indicated at the start, $S_{o} C_{s t}$, and SoC calculated by means of the overpotential and EMF models (see section 3), $S_{o} C_{l}$, of the experiment is given in columns two and three, respectively. Columns four, five and six denote the remaining run-time in minutes at the start $t_{r s t}$ of the experiment, the error in the remaining run-time $t_{r e}$ at the end of the experiment and the relative error in the remaining run-time $t_{\text {rre }}$, respectively. The remaining run-time error, $t_{r e}$, is equal to the remaining run-time value calculated by the real-time SoC evaluation system at the $3 \mathrm{~V}$ End-of-Discharge voltage level. The relative error in the remaining run-time has been defined as follows

$$
t_{r r e}[\%]=100 \frac{t_{r e}}{t_{s t}-t_{r e}}
$$

Table 2. Results retrieved with the real-time SoC evaluation system.

\begin{tabular}{|c|c|c|c|c|c|}
\hline C-rate & $\begin{array}{c}\mathrm{SoC}_{\text {st }} \\
{[\%]}\end{array}$ & $\begin{array}{c}\mathrm{SoC}_{1} \\
{[\%]}\end{array}$ & $\begin{array}{c}\mathrm{t}_{\mathrm{rst}} \\
{[\mathrm{min}]}\end{array}$ & $\begin{array}{c}\mathrm{t}_{\mathrm{re}} \\
{[\mathrm{min}]}\end{array}$ & $\begin{array}{c}\mathrm{t}_{\text {rre }} \\
{[\%]}\end{array}$ \\
\hline Measurement results retrieved without adaptation \\
\hline 0.50 & 98.3 & 3.4 & 121.8 & 7.1 & 6.2 \\
\hline 0.75 & 98.3 & 4.1 & 80.6 & 5.6 & 7.5 \\
\hline Measurement results retrieved with adaptation \\
\hline 0.10 & 98.2 & 2.7 & 577.2 & -18.0 & \\
\hline 0.25 & 98.2 & 3.2 & 229.7 & -3.6 & -1.5 \\
\hline 0.50 & 98.2 & 4.0 & 113.9 & -0.5 & -0.4 \\
\hline 0.75 & 98.2 & 4.9 & 75.2 & 0.3 & 0.4 \\
\hline 1.00 & 98.2 & 6.0 & 55.7 & 0.6 & 1.1 \\
\hline
\end{tabular}

It follows from Table 2 that when using the SoC algorithm without an adaptive system a relative error in the remaining run-time of $6.2 \%$ and $7.5 \%$ is retrieved for the discharge 0.5 and $0.75 \mathrm{C}$-rate currents, respectively. In these situations the SoC indicator makes an optimistic estimation. For instance, at the start of discharge performed at a 0.5 C-rate current, the system indicated 121.8 minutes remaining run-time. After 114.7 minutes the battery reached the level of $3 \mathrm{~V}$. This means that $t_{r r e}$ inaccuracy calculated from Eq. (7) is $6.2 \%$. In a second considered example the adaptive methods described in section 5 are used in order to adapt the maximum capacity and overpotential model parameters. It follows from Table 2 that after adaptation a $t_{\text {rre }}$ of $-0.4 \%$ and $0.4 \%$ are calculated for the discharge 0.5 and $0.75 \mathrm{C}$-rate currents. In these situations the $\mathrm{SoC}$ indicator makes slightly pessimistic and optimistic estimations. For instance, at the start of discharge performed at $0.5 \mathrm{C}$-rate current, the system indicated 113.9 minutes remaining run-time. After 114.3 minutes the battery has reached the level of 3 $\mathrm{V}$. This means that $t_{\text {rre }}$ is $-0.4 \%$. It can be concluded from the examples shown in Table 2 that a accuracy better than $3 \%$ is obtained for the remaining run-time for the adaptive system in all cases.

\section{CONCLUSIONS}

Measurement results with an adaptive State-of-Charge system have been presented in this paper. The system implementation has been particularly discussed for a mobile phone application. 
However, the system concept can be extended to other applications, e.g. electrical vehicles. The system's estimations have been calculated in each state in the form of a value of SoC and also in the form of a remaining run-time available under the valid discharge conditions. The measurements presented in this paper have been carried out with US18500G3 Li-ion batteries (Sony)

Aging of the battery is a complex process that involves many parameters of the battery such as impedance and capacity. In order to enable a much more accurate SoC indication a new adaptive system has been developed. In this system the stable conditions of the battery charging are exploited in order to adapt both $Q_{\max }$ and the overpotential parameters. In the proposed method the overpotential symmetry has been introduced in the adaptive system. Significant improvements in the remaining run-time accuracy have been obtained by using the new adaptive system. The prediction of the remaining run-time was always better than $3 \%$. It can be concluded that the newly developed adaptive system has significantly improved the accuracy of the SoC prediction.

\section{REFERENCES}

[1] H. J. Bergveld, W. S. Kruijt, P. H. L. Notten, Battery Management Systems. Design by Modelling, Philips Research Book Series, vol. 1, Kluwer Academic Publishers, Boston, 2002

[2] V. Pop, H.J. Bergveld, P.H.L. Notten, P.P.L. Regtien, A Real-Time Evaluation System for a State-of-Charge Indication Algorithm, Proceedings of the Joint International IMEKO TC1+ TC7 Symposium, vol. 1, pp. 104-107, 2005

[3] V. Pop, H.J. Bergveld, P.H.L. Notten, P.P.L. Regtien, Smart and Accurate State-of-Charge Indication in Portable Applications, IEEE Power Electronics and Drive Systems, vol. 1, pp. 262-267, 2005

[4] V. Pop, H.J. Bergveld, P.H.L. Notten, P.P.L. Regtien, State-of-the-art of state-of-charge determination, Measurement Science and Technology Journal, vol. 16, pp R93-R110, December, 2005

[5] H. J. Bergueld, V. Pop, P.H.L. Notten, Method of estimating the State-of-Charge and of the use time left of a rechargeable battery, and apparatus for executing such a method, Patent WO2005085889, filed February 23, 2005

[6] J.H. Aylor, A. Thieme, B.W. Johnson, A battery State-of-Charge Indicator for Electric Wheelchairs, IEEE Trans. On industrial electronics, vol. 39, no. 5, pp.398-409, October 1992

[7] G.R. Seyfang, Battery state of charge indicator, US Patent 4,949,046, filed June 21, 1988

[8] V. Pop, H.J. Bergveld, J.H.G. Op het Veld, P.P.L. Regtien, D. Danilov, P.H.L. Notten, Modelling battery behaviour for accurate State-of-Charge indication, J. Electrochem. Soc., 153, 11, 2006
[9] V. Pop, Universal State-of-Charge Indication for Portable Applications, Ph.D. thesis, University of Twente, 2007

\section{BIOGRAPHIES}

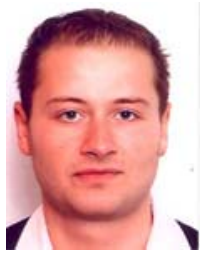

V. Pop received the M.Sc. (graduated with 10) degree in Electronics and Communications in 2002. In 2002 he joined the Measurement and Instrumentation group at the University of Twente, Enschede, the Netherlands as Ph.D. student. This research resulted in his Ph.D. degree and thesis Universal State-of-Charge Indication for Portable Applications. In September 2006 he joined the Holst Research Centre in Eindhoven, as research scientist in the Micropower Systems group.

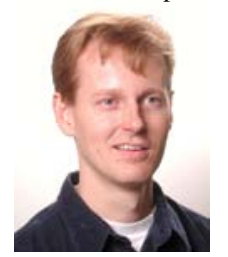

H.J. Bergveld received the M.Sc. degree (cum laude) and the Ph.D. degree (cum laude) in electrical engineering from the University of Twente, in 1994 and 2001, respectively. He joined Philips Research Laboratories, Eindhoven, the Netherlands, in 1994. His research interest was modelling of rechargeable batteries to design better battery management systems. This work resulted in his Ph.D. degree and the book Battery Management Systems - Design by modelling (Boston, MA: Kluwer, 2002). He is currently a Senior Scientist focusing on integrated DC/DC converters.

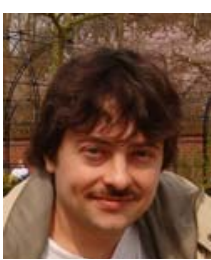

D. Danilov has a background in Physics and Mathematics and obtained his M.Sc. at the Saint-Petersburg University in 1993. In 2003 he obtained the Ph.D degree at the University of Tilburg, the Netherlands. Since then he has been working as Post-Doctoral Research Fellow at the Eurandom Institute. He conducts research on aging and State-of-Charge indication for $\mathrm{Li}$-ion rechargeable batteries. His current research interests include mathematical simulation of complex electrochemical systems, including Li-ion batteries and hydrogen storage.

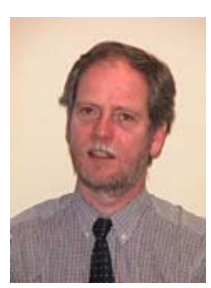

P.H.L. Notten was educated in analytical chemistry and joined the Philips Research Laboratories in 1975. While working at these laboratories on the electrochemistry of the etching of III-V semiconductors he received his Ph.D. from the Eindhoven University of Technology in 1989 and has been appointed as part-time professor at the same University in 2000 where he is heading the group Electrochemical Energy Storage, Within the Philips Research he is responsible for the research programme Portable Energy. His main interest includes modelling of various rechargeable battery systems and battery materials research.

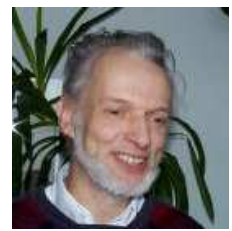

P.P.L. Regtien received the M.Sc. degree in 1970 and the Ph.D. degree in 1981, both from the Delft University of Technology, The Netherlands. Since 1994 he is a full professor at the Twente University, Department of Electrical Engineering, The Netherlands, and head of the Laboratory for Measurement and Instrumentation. Present research activities are measurement science, imaging (optical, acoustic, tactile), mechatronics, sensor technology and instrumentation. He is member of the IoP (UK), Senior member of the IEEE, board member of the IoP journal Measurement Science and Technology, the IMEKO journal Measurement and the IEEE Sensors Journal. 